\title{
Localization Principle for Triebel-Lizorkin Spaces on Spaces of Homogeneous Type
}

\author{
Dachun YANG \\ Department of Mathematics \\ Beijing Normal University \\ Beijing 100875 \\ People's Republic of China \\ dcyang@bnu.edu.cn
}

Recibido: 6 de Mayo de 2002

Aceptado: 24 de Diciembre de 2003

\section{ABSTRACT}

The author establishes the localization principle for the Triebel-Lizorkin spaces on spaces of homogeneous type.

Key words: space of homogeneous type, Triebel-Lizorkin space, localization principle, atom, Littlewood-Paley $S$-function, Calderón-Zygmund reproducing formula

2000 Mathematics Subject Classification: Primary 43A85; Secondary 46E35

\section{Introduction}

One has known that spaces of homogeneous type introduced by Coifman and Weiss in [2] include the Euclidean space, the $n$-torus in $\mathbb{R}^{n}$, the $C^{\infty}$-compact Riemannian manifolds, the boundaries of Lipschitz domains and, in particular, the Lipschitz manifolds introduced recently by Triebel in [17] and the $d$-sets in $\mathbb{R}^{n}$. It has been proved by Triebel in [15] that the $d$-sets in $\mathbb{R}^{n}$ include various kinds of fractals; see also [16]. Recently, some new Besov and Triebel-Lizorkin spaces, their characterizations and their applications were given in $[11,12]$ and [18]. In particular, in [18], it was proved that the Besov spaces on $d$-sets introduced by Triebel via traces in [15] and, equivalently, via quarkonial decompositions in [16] are the same as those Besov spaces introduced in [7] by regarding $d$-sets as special spaces of homogeneous type.

The main purpose of this paper is to establish the localization principle for TriebelLizorkin spaces on spaces of homogeneous type. Such localization principle for these spaces on $\mathbb{R}^{n}$ is well-known; see [14, p. 124]. This localization principle was proved 
to be very useful; see [14] and [17]. In fact, in [14, p. 286] and [17], this property was taken to introduce the Triebel-Lizorkin spaces on $C^{\infty}$-Riemannian manifolds, Lie groups and Lipschitz manifolds.

Let us now recall some definitions and notation on spaces of homogeneous type. A quasi-metric $\rho$ on a set $X$ is a function $\rho: X \times X \rightarrow[0, \infty)$ satisfying

(i) $\rho(x, y)=0$ if and only if $x=y$;

(ii) $\rho(x, y)=\rho(y, x)$ for all $x, y \in X$;

(iii) There exists a constant $A \in[1, \infty)$ such that for all $x, y$ and $z \in X$,

$$
\rho(x, y) \leq A[\rho(x, z)+\rho(z, y)] .
$$

Any quasi-metric defines a topology, for which the balls

$$
B(x, r)=\{y \in X: \rho(y, x)<r\}
$$

for all $x \in X$ and all $r>0$ form a basis.

The spaces of homogeneous type defined below, which was first introduced in [11], are the variants of the spaces of homogeneous type introduced by Coifman and Weiss in [2]. In what follows, we set

$$
\operatorname{diam} X=\sup \{\rho(x, y): x, y \in X\},
$$

and $A \sim B$ means that there is a constant $C>0$ independent of the main parameters such that $C^{-1}<A / B<C$. Throughout the paper, we will denote by $C$ a positive constant which is independent of the main parameters, but it may vary from line to line. Constants with subscripts, such as $C_{1}$, do not change in different occurrences. We denote $\mathbb{N} \cup\{0\}$ simply by $\mathbb{Z}_{+}$and for any $q \in[1, \infty]$, we denote by $q^{\prime}$ its conjugate index, namely, $1 / q+1 / q^{\prime}=1$.

Definition 1.1. Let $d>0$ and $\theta \in(0,1]$. A space of homogeneous type $(X, \rho, \mu)_{d, \theta}$ is a set $X$ together with a quasi-metric $\rho$ and a nonnegative Borel regular measure $\mu$ on $X$ with $\operatorname{supp} \mu=X$ and there exists a constant $C>0$ such that for all $0<r<\operatorname{diam} X$ and all $x, x^{\prime}, y \in X$,

$$
\mu(B(x, r)) \sim r^{d}
$$

and

$$
\left|\rho(x, y)-\rho\left(x^{\prime}, y\right)\right| \leq C \rho\left(x, x^{\prime}\right)^{\theta}\left[\rho(x, y)+\rho\left(x^{\prime}, y\right)\right]^{1-\theta} .
$$

Throughout this paper, we have no restriction on $\operatorname{diam} X$. In particular, when $\operatorname{diam} X<\infty$, spaces of homogeneous type appearing in Definition 1.1 cover bounded Lipschitz domains and their boundaries, Lipschitz manifolds of compact case in [17], and compact $d$-sets which include various kinds of fractals; see [15], [16] and [18]; while 
when $\operatorname{diam} X=\infty$, spaces of homogeneous type in Definition 1.1 specifically include Euclidean spaces and Lipschitz manifolds of non-compact case in [17]. Moreover, in Definition 1.1, if we choose $d=1$, then Macias and Segovia in [13] have proved that, in the sense of topological equivalence, the spaces $(X, \rho, \mu)_{d, \theta}$ are just the spaces of homogeneous type in the sense of Coifman and Weiss, whose definitions only require that $\rho$ is a quasi-metric without (2) and $\mu$ satisfies the following doubling condition which is weaker than (1): there is a constant $A^{\prime}>0$ such that for all $x \in X$ and all $r>0$,

$$
\mu(B(x, 2 r)) \leq A^{\prime} \mu(B(x, r)) .
$$

We now recall the definition of the spaces of test functions on $X$ in [10]; see also [6].

Definition 1.2. Fix $\gamma>0$ and $\theta \geq \beta>0$. A function $f$ defined on $X$ is said to be a test function of type $\left(x_{0}, r, \beta, \gamma\right)$ with $x_{0} \in X$ and $r>0$, if $f$ satisfies the following conditions:

(i) $|f(x)| \leq C \frac{r^{\gamma}}{\left(r+\rho\left(x, x_{0}\right)\right)^{d+\gamma}}$;

(ii) $|f(x)-f(y)| \leq C\left(\frac{\rho(x, y)}{r+\rho\left(x, x_{0}\right)}\right)^{\beta} \frac{r^{\gamma}}{\left(r+\rho\left(x, x_{0}\right)\right)^{d+\gamma}}$

$$
\text { for } \rho(x, y) \leq \frac{1}{2 A}\left[r+\rho\left(x, x_{0}\right)\right] \text {. }
$$

If $f$ is a test function of type $\left(x_{0}, r, \beta, \gamma\right)$, we write $f \in \mathcal{G}\left(x_{0}, r, \beta, \gamma\right)$, and the norm of $f$ in $\mathcal{G}\left(x_{0}, r, \beta, \gamma\right)$ is defined by

$$
\|f\|_{\mathcal{G}\left(x_{0}, r, \beta, \gamma\right)}=\inf \{C:(\mathrm{i}) \text { and (ii) hold }\} .
$$

Here and in what follows, $\theta$ is the same as in (2).

Now fix $x_{0} \in X$ and let $\mathcal{G}(\beta, \gamma)=\mathcal{G}\left(x_{0}, 1, \beta, \gamma\right)$. It is easy to see that

$$
\mathcal{G}\left(x_{1}, r, \beta, \gamma\right)=\mathcal{G}(\beta, \gamma)
$$

with the equivalent norms for all $x_{1} \in X$ and $r>0$. Furthermore, it is easy to check that $\mathcal{G}(\beta, \gamma)$ is a Banach space with respect to the norm in $\mathcal{G}(\beta, \gamma)$. Also, let the dual space $(\mathcal{G}(\beta, \gamma))^{\prime}$ be all linear functionals $\mathcal{L}$ from $\mathcal{G}(\beta, \gamma)$ to $\mathbb{C}$ with the property that there exists a finite constant $C \geq 0$ such that for all $f \in \mathcal{G}(\beta, \gamma)$,

$$
|\mathcal{L}(f)| \leq C\|f\|_{\mathcal{G}(\beta, \gamma)} .
$$

We denote by $\langle h, f\rangle$ the natural pairing of elements $h \in(\mathcal{G}(\beta, \gamma))^{\prime}$ and $f \in \mathcal{G}(\beta, \gamma)$. It is also easy to see that for all $h \in(\mathcal{G}(\beta, \gamma))^{\prime},\langle h, f\rangle$ is well defined for all $f \in$ $\mathcal{G}\left(x_{0}, r, \beta, \gamma\right)$ with $x_{0} \in X$ and $r>0$. Moreover, in what follows, we will denote by $\stackrel{\mathcal{G}}{(} \beta, \gamma)$, for $0<\beta, \gamma<\theta$, the completion of $\mathcal{G}(\theta, \theta)$ in $\mathcal{G}(\beta, \gamma)$. 
To state the definition of the inhomogeneous Triebel-Lizorkin spaces $F_{p q}^{s}(X)$ studied in [7], we need the following approximations to the identity which were first introduced in [6].

Definition 1.3. A sequence $\left\{S_{k}\right\}_{k=0}^{\infty}$ of linear operators is said to be an approximation to the identity of order $\epsilon \in(0, \theta]$ if there exist $C_{1}, C_{2}>0$ such that for all $k \in \mathbb{Z}_{+}$ and all $x, x^{\prime}, y$ and $y^{\prime} \in X, S_{k}(x, y)$, the kernel of $S_{k}$ is a function from $X \times X$ into $\mathbb{C}$ satisfying

(i) $S_{k}(x, y)=0$ if $\rho(x, y) \geq C_{1} 2^{-k}$ and $\left\|S_{k}\right\|_{L^{\infty}(X \times X)} \leq C_{2} 2^{d k}$;

(ii) $\left|S_{k}(x, y)-S_{k}\left(x^{\prime}, y\right)\right| \leq C_{2} 2^{k(d+\epsilon)} \rho\left(x, x^{\prime}\right)^{\epsilon}$;

(iii) $\left|S_{k}(x, y)-S_{k}\left(x, y^{\prime}\right)\right| \leq C_{2} 2^{k(d+\epsilon)} \rho\left(y, y^{\prime}\right)^{\epsilon}$;

(iv) $\left|\left[S_{k}(x, y)-S_{k}\left(x, y^{\prime}\right)\right]-\left[S_{k}\left(x^{\prime}, y\right)-S_{k}\left(x^{\prime}, y^{\prime}\right)\right]\right| \leq C_{2} 2^{k(d+2 \epsilon)} \rho\left(x, x^{\prime}\right)^{\epsilon} \rho\left(y, y^{\prime}\right)^{\epsilon}$;

(v) $\int_{X} S_{k}(x, y) d \mu(y)=1$;

(vi) $\int_{X} S_{k}(x, y) d \mu(x)=1$.

Here, that $S_{k}(x, y)$ is the kernel of $S_{k}$ means that for suitable functions $f$,

$$
S_{k} f(x)=\int_{X} S_{k}(x, y) f(y) d \mu(y)
$$

We point that by a similar Coifman's construction to that in [3], one can construct an approximation to the identity with compact supports as in Definition 1.3 for those spaces of homogeneous type in Definition 1.1.

We also need the following construction of Christ in [1], which provides an analogue of the grid of Euclidean dyadic cubes on a space of homogeneous type.

Lemma 1.4. Let $(X, \rho, \mu)_{d, \theta}$ be a space of homogeneous type. Then there exists a collection $\left\{Q_{\alpha}^{k} \subset X: k \in \mathbb{Z}_{+}, \alpha \in I_{k}\right\}$ of open subsets, where $I_{k}$ is some (possibly finite) index set, and constants $\delta \in(0,1), a_{0}>0$ and $C_{3}>0$ such that

(i) $\mu\left(X \backslash \cup_{\alpha} Q_{\alpha}^{k}\right)=0$ for each fixed $k$ and $Q_{\beta}^{k} \cap Q_{\alpha}^{k}=\emptyset$ if $\alpha \neq \beta$;

(ii) for any $\alpha, \beta, k, l$ with $l \geq k$, either $Q_{\beta}^{l} \subset Q_{\alpha}^{k}$ or $Q_{\beta}^{l} \cap Q_{\alpha}^{k}=\emptyset$;

(iii) for each $(k, \alpha)$ and each $l<k$ there is a unique $\beta$ such that $Q_{\alpha}^{k} \subset Q_{\beta}^{l}$;

(iv) $\operatorname{diam} Q_{\alpha}^{k} \leq C_{3} \delta^{k}$;

(v) each $Q_{\alpha}^{k}$ contains some ball $B\left(z_{\alpha}^{k}, a_{0} \delta^{k}\right)$, where $z_{\alpha}^{k} \in X$. 
In fact, we can think of $Q_{\alpha}^{k}$ as being essentially a cube of diameter rough $\delta^{k}$ with center $z_{\alpha}^{k}$. In what follows, we always suppose $\delta=1 / 2$. See [10] for how to remove this restriction. Also, we will denote by $Q_{\tau}^{k, \nu}, \nu=1,2, \ldots, N(k, \tau)$, the set of all cubes $Q_{\tau^{\prime}}^{k+j} \subset Q_{\tau}^{k}$, where $j$ is a fixed large positive integer. Denote by $y_{\tau}^{k, \nu}$ a point in $Q_{\tau}^{k, \nu}$. For any dyadic cube $Q$ and any $f \in L_{\text {loc }}^{1}(X)$, we set

$$
m_{Q}(f)=\frac{1}{\mu(Q)} \int_{Q} f(x) d \mu(x),
$$

and we also let $a_{+}=\max (a, 0)$ for any $a \in \mathbb{R}$.

Now we can state the definition of the Triebel-Lizorkin spaces $F_{p q}^{s}(X)$ in [12].

Definition 1.5. Let $s \in(-\theta, \theta),\left\{S_{k}\right\}_{k=0}^{\infty}$ be as in Definition 1.3 with order $\theta, D_{0}=S_{0}$ and $D_{k}=S_{k}-S_{k-1}$ for $k \in \mathbb{N}$. Suppose $\beta$ and $\gamma$ satisfying

$$
\max \left(0,-s+d(1 / p-1)_{+}\right)<\beta<\theta \quad \text { and } \quad 0<\gamma<\theta .
$$

Let $j \in \mathbb{N}$ be fixed and large enough and $\left\{Q_{\tau}^{0, \nu}: \tau \in I_{0}, \nu=1, \ldots, N(0, \tau)\right\}$ be as above. The inhomogeneous Triebel-Lizorkin space $F_{p q}^{s}(X)$ for

$$
\max (d /(d+\theta), d /(d+\theta+s))<p<\infty
$$

and $\max (d /(d+\theta), d /(d+\theta+s))<q \leq \infty$ is the collection of all $f \in(\dot{\mathcal{G}}(\beta, \gamma))^{\prime}$ such that

$$
\begin{aligned}
\|f\|_{F_{p q}^{s}(X)}=\left\{\sum_{\tau \in I_{0}} \sum_{\nu=1}^{N(0, \tau)} \mu\left(Q_{\tau}^{0, \nu}\right)\left[m_{Q_{\tau}^{0, \nu}}\left(\left|D_{0}(f)\right|\right)\right]^{p}\right\}^{1 / p} \\
+\left\|\left\{\sum_{k=1}^{\infty}\left[2^{k s}\left|D_{k}(f)\right|\right]^{q}\right\}^{1 / q}\right\|_{L^{p}(X)}<\infty .
\end{aligned}
$$

It was proved in [12] that Definition 1.5 is independent of the choices of large positive integers $j$, approximations to the identity and the pairs $(\beta, \gamma)$ as in (3).

To state our main theorem, we need a resolution of unity $\left\{\psi_{\alpha}\right\}_{\alpha}$ on $X$ satisfying:

(a) $0 \leq \psi_{\alpha} \leq 1, \operatorname{supp} \psi_{\alpha} \subset B\left(z_{\alpha}, C_{4}\right)=\left\{x \in X: \rho\left(x, z_{\alpha}\right)<C_{4}\right\}, C_{4}>0$ is a constant and

$$
B\left(z_{\alpha}, C_{4} / 2\right) \cap B\left(z_{\beta}, C_{4} / 2\right)=\emptyset \text { if } \neq \beta ;
$$

(b) Let $U=\cup_{\alpha} B\left(z_{\alpha}, C_{4} / 2\right)$, then $\mu(X \backslash U)=0$ and $\psi_{\alpha} \in \operatorname{Lip}_{\theta}(U)$ with a uniform Lip constant in $\alpha$;

(c) $\sum_{\alpha} \psi_{\alpha}(x)=1$ on $U$. 
We will show the existence of such a resolution of unity in the next section. Here is our main theorem.

Theorem 1.6. Let $\left\{\psi_{\alpha}\right\}_{\alpha}$ satisfy (a), (b) and (c) as above. Let $s \in(-\theta, \theta)$, $\max (d /(d+\theta), d /(d+\theta+s))<p<\infty$ and $\max (d /(d+\theta), d /(d+\theta+s))<q \leq \infty$, then

$$
\left\{\sum_{\alpha}\left\|\psi_{\alpha} f\right\|_{F_{p q}^{s}(X)}^{p}\right\}^{1 / p}
$$

is an equivalent quasi-norm in $F_{p q}^{s}(X)$.

The proof of Theorem 1.6 will be given in the next section. We remark that our proof is essentially different from the proof of Theorem 2.4.7 in [14] on the localization principle for the Triebel-Lizorkin spaces on $\mathbb{R}^{n}$, since there is no theory of Fourier transforms and differentiations on spaces of homogeneous type. The new ingredient in our proof is the applications of the inhomogeneous Calderón reproducing formulae in $[9]$.

Note that $\mathbb{R}^{n}$ with the Lebesgue measure and the standard Euclidean metric $|\cdot|$ is a space of homogeneous type in the sense of Definition 1.1, that is, the homogeneous space, $\left(\mathbb{R}^{n},|\cdot|, m\right)_{n, 1}$; see [11]. By this fact and Theorem 2.4.7 in [14], one can not expect that we have the localization principle for the Besov spaces $B_{p q}^{s}(X)$ similar to Theorem 1.6. See [14] for more details.

\section{Proof of Theorem 1.6}

Let us first show the existence of resolutions of unity satisfying (a), (b) and (c). To do so, we need to use Lemma 1.4, (i).

Let $U=\cup_{\alpha} Q_{\alpha}^{0}$, then $\mu(X \backslash U)=0$ by Lemma 1.4, (i). We now choose a function $\varphi \in C^{\infty}(\mathbb{R}), \varphi \geq 0, \operatorname{supp} \varphi \subset\left(0,2 C_{3}\right)$ and $\varphi(x)=1$ if $x \in\left(0, C_{3}\right)$. Let $\varphi_{\alpha}=$ $\varphi\left(\rho\left(x, z_{\alpha}^{0}\right)\right)$ and for $x \in U$, let

$$
\psi_{\alpha}(x)=\frac{\varphi_{\alpha}(x)}{\sum_{\alpha} \varphi_{\alpha}(x)} .
$$

Then it is easy to see that $\left\{\psi_{\alpha}\right\}_{\alpha}$ satisfies (a), (b) and (c).

As pointed above, one important tool for us to show Theorem 1.6 is the following inhomogeneous Calderón reproducing formulae in [9].

Lemma 2.1. Suppose that $\left\{D_{k}\right\}_{k=0}^{\infty}$ is the same as in Definition 1.5. Then there exist functions $\widetilde{D}_{Q_{\tau}^{0, \nu}}, \tau \in I_{0}$ and $\nu=1, \ldots, N(0, \tau)$, and $\widetilde{D}_{k}(x, y), k \in \mathbb{N}$, such that for any fixed $y_{\tau}^{k, \nu} \in Q_{\tau}^{k, \nu}, k \in \mathbb{N}, \tau \in I_{k}$ and $\nu \in\{1, \ldots, N(k, \tau)\}$ and all $f \in\left(\mathcal{G}\left(\beta_{1}, \gamma_{1}\right)\right)^{\prime}$ 
with $0<\beta_{1}<\theta$ and $0<\gamma_{1}<\theta$,

$$
\begin{aligned}
f(x)=\sum_{\tau \in I_{0}} & \sum_{\nu=1}^{N(0, \tau)} \mu\left(Q_{\tau}^{0, \nu}\right) m_{Q_{\tau}^{0, \nu}}\left(D_{0}(f)\right) \widetilde{D}_{Q_{\tau}^{0, \nu}}(x) \\
& \quad+\sum_{k=1}^{\infty} \sum_{\tau \in I_{k}} \sum_{\nu=1}^{N(k, \tau)} \mu\left(Q_{\tau}^{k, \nu}\right) D_{k}(f)\left(y_{\tau}^{k, \nu}\right) \widetilde{D}_{k}\left(x, y_{\tau}^{k, \nu}\right),
\end{aligned}
$$

where the series converge in $\left(\mathcal{G}\left(\beta_{1}^{\prime}, \gamma_{1}^{\prime}\right)\right)^{\prime}$ for $\beta_{1}<\beta_{1}^{\prime}<\theta$ and $\gamma_{1}<\gamma_{1}^{\prime}<\theta$; for any given $\epsilon \in(0, \theta), \widetilde{D}_{k}(x, y), k \in \mathbb{N}$, satisfies that

(i) $\left|\widetilde{D}_{k}(x, y)\right| \leq C \frac{2^{-k \epsilon}}{\left(2^{-k}+\rho(x, y)\right)^{d+\epsilon}}$,

(ii) $\left|\widetilde{D}_{k}(x, y)-\widetilde{D}_{k}\left(x^{\prime}, y\right)\right| \leq C\left(\frac{\rho\left(x, x^{\prime}\right)}{2^{-k}+\rho(x, y)}\right)^{\epsilon} \frac{2^{-k \epsilon}}{\left(2^{-k}+\rho(x, y)\right)^{d+\epsilon}}$

$$
\text { for } \rho\left(x, x^{\prime}\right) \leq \frac{1}{2 A}\left(2^{-k}+\rho(x, y)\right) \text {, }
$$

(iii) $\int_{X} \widetilde{D}_{k}(x, y) d \mu(x)=\int_{X} \widetilde{D}_{k}(x, y) d \mu(y)=0$;

$\operatorname{diam}\left(Q_{\tau}^{0, \nu}\right) \sim 2^{-j}$ for $\tau \in I_{0}$ and $\nu=1, \ldots, N(0, \tau)$ for some $j \in \mathbb{N} ; \widetilde{D}_{Q_{\tau}^{0, \nu}}(x)$ for $\tau \in I_{0}$ and $\nu=1, \ldots, N(0, \tau)$ satisfies that

(iv) $\int_{X} \widetilde{D}_{Q_{\tau}^{0, \nu}}(x) d \mu(x)=1$,

(v) for any given $\epsilon \in(0, \theta)$, there is a constant $C>0$ such that

$$
\left|\widetilde{D}_{Q_{\tau}^{0, \nu}}(x)\right| \leq C \frac{1}{(1+\rho(x, y))^{d+\epsilon}}
$$

for all $x \in X$ and $y \in Q_{\tau}^{0, \nu}$ and

(vi)

$$
\begin{array}{r}
\left|\widetilde{D}_{Q_{\tau}^{0, \nu}}(x)-\widetilde{D}_{Q_{\tau}^{0, \nu}}(z)\right| \leq C\left(\frac{\rho(x, z)}{1+\rho(x, y)}\right)^{\epsilon} \frac{1}{(1+\rho(x, y))^{d+\epsilon}} \\
\text { for all } x, z \in X \text { and all } y \in Q_{\tau}^{0, \nu} \text { satisfying } \rho(x, z) \leq \frac{1}{2 A}(1+\rho(x, y)) .
\end{array}
$$

Moreover, $j$ can be any fixed large positive integer and the constant $C$ in (i), (ii), (v) and (vi) is independent of $j$.

We also need the following lemma which can be found in [5, pp. 147-148] for $\mathbb{R}^{n}$ and [10, pp. 93] for spaces of homogeneous type. 
Lemma 2.2. Let $0<r \leq 1, k, \eta \in \mathbb{Z}$ with $\eta \leq k$ and for any dyadic cube $Q_{\tau}^{k, \nu}$,

$$
\left|f_{Q_{\tau}^{k, \nu}}(x)\right| \leq\left(1+2^{\eta} \rho\left(x, y_{\tau}^{k, \nu}\right)\right)^{-d-\gamma},
$$

where $y_{\tau}^{k, \nu}$ is any point in $Q_{\tau}^{k, \nu}$ and $\gamma>d(1 / r-1)$. Then

$$
\sum_{\tau \in I_{k}} \sum_{\nu=1}^{N(k, \tau)}\left|\lambda_{Q_{\tau}^{k, \nu}}\right|\left|f_{Q_{\tau}^{k, \nu}}(x)\right| \leq C 2^{(k-\eta) d / r}\left[M\left(\sum_{\tau \in I_{k}} \sum_{\nu=1}^{N(k, \tau)}\left|\lambda_{Q_{\tau}^{k, \nu}}\right|^{r} \chi_{Q_{\tau}^{k, \nu}}\right)(x)\right]^{1 / r},
$$

where $C$ is independent of $x, k$ and $\eta$, and $M$ is the Hardy-Littlewood maximal operator on $X$.

Now we can prove Theorem 1.6.

Proof of Theorem 1.6. We first prove that

$$
\|f\|_{F_{p q}^{s}(X)} \leq C\left\{\sum_{\alpha}\left\|\psi_{\alpha} f\right\|_{F_{p q}^{s}(X)}^{p}\right\}^{1 / p} .
$$

Let $\left\{D_{k}\right\}_{k=0}^{\infty}$ be as in Definition 1.5. By (c), (b) and Definition 1.5, we have

$$
\begin{aligned}
& \left\{\sum_{\tau \in I_{0}} \sum_{\nu=1}^{N(0, \tau)} \mu\left(Q_{\tau}^{0, \nu}\right)\left[m_{Q_{\tau}^{0, \nu}}\left(\left|D_{0}(f)\right|\right)\right]^{p}\right\}^{1 / p} \\
& \leq\left\{\sum_{\tau \in I_{0}} \sum_{\nu=1}^{N(0, \tau)} \mu\left(Q_{\tau}^{0, \nu}\right)\left[\sum_{\alpha} m_{Q_{\tau}^{0, \nu}}\left(\left|D_{0}(f)\right|\right)\right]^{p}\right\}^{1 / p} \\
& \leq\left\{\sum_{\tau \in I_{0}} \sum_{\nu=1}^{N(0, \tau)} \mu\left(Q_{\tau}^{0, \nu}\right) \sum_{\alpha}\left[m_{Q_{\tau}^{0, \nu}}\left(\left|D_{0}(f)\right|\right)\right]^{p}\right\}^{1 / p} \\
& \leq C\left\{\sum_{\alpha}\left\|\psi_{\alpha} f\right\|_{F_{p q}^{s}(X)}^{p}\right\}^{1 / p},
\end{aligned}
$$

where, to get the second-to-last inequality, when $p \leq 1$, we have used the following inequality:

$$
\left(\sum_{\alpha}\left|a_{\alpha}\right|\right)^{p} \leq \sum_{\alpha}\left|a_{\alpha}\right|^{p}
$$

for all $a_{\alpha} \in \mathbb{C}$; while when $1<p<\infty$, we have used the following fact: noting that $\operatorname{supp} D_{0}\left(\psi_{\alpha} f\right) \subset\left\{x: \rho\left(x, z_{\alpha}\right) \leq A\left(3 C_{1}+C_{4}\right)\right\}$, for giving $\tau$ and $\nu$, we obtain

$$
\#\left\{\alpha: Q_{\tau}^{0, \nu} \cap B\left(z_{\alpha}, A\left(3 C_{1}+C_{4}\right) \neq \emptyset\right\} \leq C .\right.
$$


Again, noting that for $k \in \mathbb{N}$, supp $D_{k}\left(\psi_{\alpha} f\right) \subset\left\{x: \rho\left(x, z_{\alpha}\right) \leq A\left(3 C_{1}+C_{4}\right)\right\}$ and

$$
\sum_{\alpha} \chi_{B\left(z_{\alpha}, A\left(3 C_{1}+C_{4}\right)\right)}(x) \leq C,
$$

by (c), (b) and Definition 1.5, we have

$$
\begin{gathered}
\left\|\left\{\sum_{k=1}^{\infty}\left[2^{k s}\left|D_{k}(f)\right|\right]^{q}\right\}^{1 / q}\right\|_{L^{p}(X)} \\
\leq\left\|\left\{\sum_{k=1}^{\infty}\left[2^{k s} \sum_{\alpha}\left|D_{k}\left(\psi_{\alpha} f\right)\right|\right]^{q}\right\}^{1 / q}\right\|_{L^{p}(X)} \\
\leq C\left\|\left\{\sum_{\alpha} \sum_{k=1}^{\infty}\left[2^{k s}\left|D_{k}\left(\psi_{\alpha} f\right)\right|\right]^{q}\right\}^{1 / q}\right\|_{L^{p}(X)}
\end{gathered}
$$

(when $q>1$, by (9) and the Hölder inequality; when $q \leq 1$, by (7))

$$
\leq C\left(\sum_{\alpha}\left\|\left\{\sum_{k=1}^{\infty}\left[2^{k s}\left|D_{k}\left(\psi_{\alpha} f\right)\right|\right]^{q}\right\}^{1 / q}\right\|_{L^{p}(X)}^{p}\right)^{1 / p}
$$

(when $p / q>1$, by (9) and the Hölder inequality; when $p / q \leq 1$, by (7))

$$
=\left\{\sum_{\alpha}\left\|\psi_{\alpha} f\right\|_{F_{p q}^{s}(X)}^{p}\right\}^{1 / p} .
$$

Estimates (6), (10) and Definition 1.5 imply (5).

Let us now establish the converse of (5). By Lemma 2.1, we have

$$
\begin{aligned}
D_{0}\left(\psi_{\alpha} f\right)(x)= & \int_{X} D_{0}(x, y) \psi_{\alpha}(y) f(y) d \mu(y) \\
= & \sum_{\tau^{\prime} \in I_{0}} \sum_{\nu^{\prime}=1}^{N\left(0, \tau^{\prime}\right)} \mu\left(Q_{\tau^{\prime}}^{0, \nu^{\prime}}\right) m_{Q_{\tau^{\prime}}^{0, \nu^{\prime}}}\left(D_{0}(f)\right) \int_{X} D_{0}(x, y) \psi_{\alpha}(y) \widetilde{D}_{Q_{\tau^{\prime}}^{0, \nu^{\prime}}}(y) d \mu(y) \\
& \quad+\sum_{k^{\prime}=1}^{\infty} \sum_{\tau^{\prime} \in I_{k^{\prime}}} \sum_{\nu^{\prime}=1}^{N\left(k^{\prime}, \tau^{\prime}\right)} \mu\left(Q_{\tau^{\prime}}^{k^{\prime}, \nu^{\prime}}\right) D_{k^{\prime}}(f)\left(y_{\tau^{\prime}}^{k^{\prime}, \nu^{\prime}}\right) \\
& \quad \times \int_{X} D_{0}(x, y) \psi_{\alpha}(y) \widetilde{D}_{k^{\prime}}\left(y, y_{\tau^{\prime}}^{k^{\prime}, \nu^{\prime}}\right) d \mu(y)=E_{1}+E_{2}
\end{aligned}
$$


By (a), the support of $D_{0}$ and the estimate (v) satisfied by $\widetilde{D}_{Q_{\tau^{\prime}}^{0, \nu^{\prime}}}$ in Lemma 2.1, we can estimate $E_{1}$ by

$$
\begin{aligned}
\left|E_{1}\right| \leq & C \sum_{\tau^{\prime} \in I_{0}} \sum_{\nu^{\prime}=1}^{N\left(0, \tau^{\prime}\right)} \mu\left(Q_{\tau^{\prime}}^{0, \nu^{\prime}}\right) m_{Q_{\tau^{\prime}}^{0, \nu^{\prime}}}\left(\left|D_{0}(f)\right|\right) \chi_{B\left(z_{\alpha}, A\left(C_{1}+C_{4}\right)\right)}(x) \\
& \times \frac{1}{\left(1+\rho\left(y_{\tau}^{0, \nu}, y_{\tau^{\prime}}^{0, \nu^{\prime}}\right)\right)^{d+\epsilon}} \int_{X}\left|D_{0}(x, y)\right| d \mu(y) \\
\leq & C \sum_{\tau^{\prime} \in I_{0}} \sum_{\nu^{\prime}=1}^{N\left(0, \tau^{\prime}\right)} \mu\left(Q_{\tau^{\prime}}^{0, \nu^{\prime}}\right) m_{Q_{\tau^{\prime}}^{0, \nu^{\prime}}}\left(\left|D_{0}(f)\right|\right) \chi_{B\left(z_{\alpha}, A\left(C_{1}+C_{4}\right)\right)}(x) \\
& \times \frac{1}{\left(1+\rho\left(y_{\tau}^{0, \nu}, y_{\tau^{\prime}}^{0, \nu^{\prime}}\right)\right)^{d+\epsilon}},
\end{aligned}
$$

where we choose $\epsilon>0$ such that $p>d /(d+\epsilon)$. From this, it follows that

$$
\begin{aligned}
& \left\{\sum_{\alpha} \sum_{\tau \in I_{0}} \sum_{\nu=1}^{N(0, \tau)} \mu\left(Q_{\tau}^{0, \nu}\right)\left[m_{Q_{\tau}^{0, \nu}}\left(\left|E_{1}\right|\right)\right]^{p}\right\}^{1 / p} \\
& \leq C\left\{\sum _ { \tau \in I _ { 0 } } \sum _ { \nu = 1 } ^ { N ( 0 , \tau ) } \mu ( Q _ { \tau } ^ { 0 , \nu } ) \left[\sum_{\tau^{\prime} \in I_{0}} \sum_{\nu^{\prime}=1}^{N\left(0, \tau^{\prime}\right)} \mu\left(Q_{\tau^{\prime}}^{0, \nu^{\prime}}\right) m_{Q_{\tau^{\prime}}^{0, \nu^{\prime}}}\left(\left|D_{0}(f)\right|\right)\right.\right. \\
& \left.\left.\quad \times \frac{1}{\left(1+\rho\left(y_{\tau}^{0, \nu}, y_{\tau^{\prime}}^{0, \nu^{\prime}}\right)\right)^{d+\epsilon}} \frac{1}{\mu\left(Q_{\tau}^{0, \nu}\right)} \int_{Q_{\tau}^{0, \nu}} \sum_{\alpha} \chi_{B\left(z_{\alpha}, A\left(C_{1}+C_{4}\right)\right)}(x) d \mu(x)\right]^{p}\right\}^{1 / p}
\end{aligned}
$$

(by (8))

$$
\leq C\left\{\sum _ { \tau \in I _ { 0 } } \sum _ { \nu = 1 } ^ { N ( 0 , \tau ) } \mu ( Q _ { \tau } ^ { 0 , \nu } ) \left[\sum_{\tau^{\prime} \in I_{0}} \sum_{\nu^{\prime}=1}^{N\left(0, \tau^{\prime}\right)} \mu\left(Q_{\tau^{\prime}}^{0, \nu^{\prime}}\right) m_{Q_{\tau^{\prime}}^{0, \nu^{\prime}}}\left(\left|D_{0}(f)\right|\right)\right.\right.
$$




$$
\begin{aligned}
& \left.\left.\times \frac{1}{\left(1+\rho\left(y_{\tau}^{0, \nu}, y_{\tau^{\prime}}^{0, \nu^{\prime}}\right)\right)^{d+\epsilon}}\right]^{p}\right\}^{1 / p}(\text { by }(9)) \\
& \left\{\sum _ { \tau \in I _ { 0 } } \sum _ { \nu = 1 } ^ { N ( 0 , \tau ) } \mu ( Q _ { \tau } ^ { 0 , \nu } ) \left(\sum_{\tau^{\prime} \in I_{0}} \sum_{\nu^{\prime}=1}^{N\left(0, \tau^{\prime}\right)} \mu\left(Q_{\tau^{\prime}}^{0, \nu^{\prime}}\right)\left[m_{Q_{\tau^{\prime}}^{0, \nu^{\prime}}}\left(\left|D_{0}(f)\right|\right)\right]^{p}\right.\right. \\
& \left.\left.\frac{1}{\left(1+\rho\left(y_{\tau}^{0, \nu}, y_{\tau^{\prime}}^{0, \nu^{\prime}}\right)\right)^{d+\epsilon}}\right)\left(\int_{X} \frac{1}{\left(1+\rho\left(y_{\tau}^{0, \nu}, y\right)\right)^{d+\epsilon}} d \mu(y)\right)^{p / p^{\prime}}\right\}^{1 / p}, \\
& \leq C\left\{\begin{array}{c}
1<p<\infty \quad \text { (by the Hölder } \\
\left\{\sum_{\tau^{\prime} \in I_{0}} \sum_{\nu^{\prime}=1}^{N\left(0, \tau^{\prime}\right)} \mu\left(Q_{\tau^{\prime}}^{0, \nu^{\prime}}\right)\left[m_{Q_{\tau^{\prime}}^{0, \nu^{\prime}}}\left(\left|D_{0}(f)\right|\right)\right]^{p}\right.
\end{array}\right. \\
& \left.\times \int_{X} \frac{1}{\left(1+\rho\left(x, y_{\tau^{\prime}}^{0, \nu^{\prime}}\right)\right)^{(d+\epsilon) p}} d \mu(x)\right\}^{1 / p}, p \leq 1 \\
& \text { (by (7) and } \mu\left(Q_{\tau^{\prime}}^{0, \nu^{\prime}}\right) \sim C \text { ) } \\
& \leq C\left\{\sum_{\tau^{\prime} \in I_{0}} \sum_{\nu^{\prime}=1}^{N\left(0, \tau^{\prime}\right)} \mu\left(Q_{\tau^{\prime}}^{0, \nu^{\prime}}\right)\left[m_{Q_{\tau^{\prime}}^{0, \nu^{\prime}}}\left(\left|D_{0}(f)\right|\right)\right]^{p}\right\}^{1 / p} \leq C\|f\|_{F_{p q}^{s}(X)} .
\end{aligned}
$$

By the supports of $D_{0}$ and $\psi_{\alpha}$ and the vanishing moment satisfied by $\widetilde{D}_{k^{\prime}}$, we can estimate $E_{2}$ by

$$
\begin{aligned}
\left|E_{2}\right|= & \left|\sum_{k^{\prime}=1}^{\infty} \sum_{\tau^{\prime} \in I_{k^{\prime}}} \sum_{\nu^{\prime}=1}^{N(k, \tau)} \mu\left(Q_{\tau^{\prime}}^{k^{\prime}, \nu^{\prime}}\right) D_{k^{\prime}}(f)\left(y_{\tau^{\prime}}^{k^{\prime}, \nu^{\prime}}\right) \int_{X} D_{0}(x, y) \psi_{\alpha}(y) \widetilde{D}_{k^{\prime}}\left(y, y_{\tau^{\prime}}^{k^{\prime}, \nu^{\prime}}\right) d \mu(y)\right| \\
\leq & \sum_{k^{\prime}=1}^{\infty} \sum_{\tau^{\prime} \in I_{k^{\prime}}} \sum_{\nu^{\prime}=1}^{N\left(k^{\prime}, \tau^{\prime}\right)} \mu\left(Q_{\tau^{\prime}}^{k^{\prime}, \nu^{\prime}}\right)\left|D_{k^{\prime}}(f)\left(y_{\tau^{\prime}}^{k^{\prime}, \nu^{\prime}}\right)\right| \chi_{B\left(z_{\alpha}, A\left(C_{1}+C_{4}\right)\right)}(x) \\
& \times\left|\int_{X}\left[D_{0}(x, y) \psi_{\alpha}(y)-D_{0}\left(x, y_{\tau^{\prime}}^{k^{\prime}, \nu^{\prime}}\right) \psi_{\alpha}\left(y_{\tau^{\prime}}^{k^{\prime}, \nu^{\prime}}\right)\right] \widetilde{D}_{k^{\prime}}\left(y, y_{\tau^{\prime}}^{k^{\prime}, \nu^{\prime}}\right) d \mu(y)\right| \\
\leq & \sum_{k^{\prime}=1}^{\infty} \sum_{\tau^{\prime} \in I_{k^{\prime}}} \sum_{\nu^{\prime}=1}^{N\left(k^{\prime}, \tau^{\prime}\right)} \mu\left(Q_{\tau^{\prime}}^{k^{\prime}, \nu^{\prime}}\right)\left|D_{k^{\prime}}(f)\left(y_{\tau^{\prime}}^{k^{\prime}, \nu^{\prime}}\right)\right| \chi_{B\left(z_{\alpha}, A\left(C_{1}+C_{4}\right)\right)}(x) \\
& \times\left[\int_{\left\{y \in X: \rho\left(y, y_{\tau^{\prime}}^{k^{\prime}, \nu^{\prime}}\right) \leq \frac{1}{2 A}\left(1+\rho\left(x, y_{\tau^{\prime}}^{k^{\prime}, \nu^{\prime}}\right)\right)\right\}} \mid\left[D_{0}(x, y) \psi_{\alpha}(y)-D_{0}\left(x, y_{\tau^{\prime}}^{k^{\prime}, \nu^{\prime}}\right) \psi_{\alpha}\left(y_{\tau^{\prime}}^{k^{\prime}, \nu^{\prime}}\right)\right]\right.
\end{aligned}
$$




$$
\begin{aligned}
& \left.\times \widetilde{D}_{k^{\prime}}\left(y, y_{\tau^{\prime}}^{k^{\prime}, \nu^{\prime}}\right) \mid d \mu(y)+\int_{\left\{y \in X: \rho\left(y, y_{\tau^{\prime}}^{k^{\prime}, \nu^{\prime}}\right)>\frac{1}{2 A}\left(1+\rho\left(x, y_{\tau^{\prime}}^{k^{\prime}, \nu^{\prime}}\right)\right)\right\}} \cdots\right] \\
= & \sum_{k^{\prime}=1}^{\infty} \sum_{\tau^{\prime} \in I_{k^{\prime}}} \sum_{\nu^{\prime}=1}^{N\left(k^{\prime}, \tau^{\prime}\right)} \mu\left(Q_{\tau^{\prime}}^{k^{\prime}, \nu^{\prime}}\right)\left|D_{k^{\prime}}(f)\left(y_{\tau^{\prime}}^{k^{\prime}, \nu^{\prime}}\right)\right| \chi_{B\left(z_{\alpha}, A\left(C_{1}+C_{4}\right)\right)}(x)\left[E_{2}^{1}+E_{2}^{2}\right] .
\end{aligned}
$$

From the support satisfied by $D_{0}$, the regularities satisfied by $D_{0}$ and $\psi_{\alpha}$ and the size condition satisfied by $\widetilde{D}_{k^{\prime}}$, it follows that

$$
\begin{aligned}
E_{2}^{1}= & \int_{\left\{y \in X: \rho\left(y, y_{\tau^{\prime}, \nu^{\prime}}^{k^{\prime}}\right) \leq \frac{1}{2 A}\left(1+\rho\left(x, y_{\tau^{\prime}}^{k^{\prime}, \nu^{\prime}}\right)\right)\right\}} \mid\left(\left[D_{0}(x, y)-D_{0}\left(x, y_{\tau^{\prime}}^{k^{\prime}, \nu^{\prime}}\right)\right] \psi_{\alpha}(y)\right. \\
& \left.+D_{0}\left(x, y_{\tau^{\prime}}^{k^{\prime}, \nu^{\prime}}\right)\left[\psi_{\alpha}(y)-\psi_{\alpha}\left(y_{\tau^{\prime}}^{k^{\prime}, \nu^{\prime}}\right)\right]\right) \widetilde{D}_{k^{\prime}}\left(y, y_{\tau^{\prime}}^{k^{\prime}, \nu^{\prime}}\right) \mid d \mu(y) \\
\leq & C \chi_{B\left(y_{\tau^{\prime}}^{\left.k^{\prime}, \nu^{\prime}, C\right)}\right.}(x) \int_{X} \rho\left(y, y_{\tau^{\prime}}^{k^{\prime}, \nu^{\prime}}\right)^{\epsilon_{1}}\left|\widetilde{D}_{k^{\prime}}\left(y, y_{\tau^{\prime}}^{k^{\prime}, \nu^{\prime}}\right)\right| d \mu(y) \\
\leq & C \chi_{B\left(y_{\tau^{\prime}}^{k^{\prime}, \nu^{\prime}}, C\right)}(x) \int_{X} \rho\left(y, y_{\tau^{\prime}}^{k^{\prime}, \nu^{\prime}}\right)^{\epsilon_{1}} \frac{2^{-k^{\prime} \epsilon}}{\left(2^{-k^{\prime}}+\rho\left(y, y_{\tau^{\prime}}^{k^{\prime}, \nu^{\prime}}\right)\right)^{d+\epsilon}} d \mu(y) \\
\leq & C 2^{-k^{\prime} \epsilon_{1}} \chi_{B\left(y_{\tau^{\prime}}^{k^{\prime}, \nu^{\prime}}, C\right)}(x) \leq C 2^{-k^{\prime} \epsilon_{1}} \frac{1}{\left(1+\rho\left(x, y_{\tau^{\prime}}^{k^{\prime}, \nu^{\prime}}\right)\right)^{d+\epsilon_{1}}},
\end{aligned}
$$

where $\epsilon \in(0, \theta)$ and $\epsilon_{1} \in(0, \epsilon)$ which will be determined later. For the same $\epsilon$ and $\epsilon_{1}$, we have

$$
\begin{aligned}
E_{2}^{2} \leq & \int_{\left\{y \in X: \rho\left(y, y_{\tau^{\prime}}^{k^{\prime}, \nu^{\prime}}\right)>\frac{1}{2 A}\left(1+\rho\left(x, y_{\tau^{\prime}}^{k^{\prime}, \nu^{\prime}}\right)\right)\right\}}\left[\left|D_{0}(x, y) \psi_{\alpha}(y)\right|\right. \\
& \left.+\left|D_{0}\left(x, y_{\tau^{\prime}}^{k^{\prime}, \nu^{\prime}}\right) \psi_{\alpha}\left(y_{\tau^{\prime}}^{k^{\prime}, \nu^{\prime}}\right)\right|\right]\left|\widetilde{D}_{k^{\prime}}\left(y, y_{\tau^{\prime}}^{k^{\prime}, \nu^{\prime}}\right)\right| d \mu(y) \\
\leq & \int_{\left\{y \in X: \rho\left(y, y_{\tau^{\prime}}^{k^{\prime}, \nu^{\prime}}\right)>\frac{1}{2 A}\left(1+\rho\left(x, y_{\tau^{\prime}}^{k^{\prime}, \nu^{\prime}}\right)\right)\right\}}\left|D_{0}(x, y)\right| \frac{2^{-k^{\prime} \epsilon}}{\left(2^{-k^{\prime}}+\rho\left(y, y_{\tau^{\prime}}^{k^{\prime}, \nu^{\prime}}\right)\right)} d \mu(y) \\
& +\chi_{B\left(y_{\tau^{\prime}}^{k^{\prime}, \nu^{\prime}}, C\right)}(x) 2^{-k^{\prime} \epsilon_{1}} \int_{X} \frac{2^{-k^{\prime}\left(\epsilon-\epsilon_{1}\right)}}{\left(2^{-k^{\prime}}+\rho\left(y, y_{\tau^{\prime}}^{k^{\prime}, \nu^{\prime}}\right)\right)^{d+\epsilon-\epsilon_{1}}} d \mu(y) \\
\leq & C 2^{-k^{\prime} \epsilon_{1}} \frac{1}{\left(1+\rho\left(x, y_{\tau^{\prime}}^{k^{\prime}, \nu^{\prime}}\right)\right)^{d+\epsilon_{1}}} .
\end{aligned}
$$


Thus,

$$
\begin{aligned}
\left|E_{2}\right| \leq & C \sum_{k^{\prime}=1}^{\infty} \sum_{\tau^{\prime} \in I_{k^{\prime}}} \sum_{\nu^{\prime}=1}^{N\left(k^{\prime}, \tau^{\prime}\right)} 2^{-k^{\prime} \epsilon_{1}} \mu\left(Q_{\tau^{\prime}}^{k^{\prime}, \nu^{\prime}}\right)\left|D_{k^{\prime}}(f)\left(y_{\tau^{\prime}}^{k^{\prime}, \nu^{\prime}}\right)\right| \chi_{B\left(z_{\alpha}, A\left(C_{1}+C_{4}\right)\right)}(x) \\
& \times \frac{1}{\left(1+\rho\left(x, y_{\tau^{\prime}}^{k^{\prime}, \nu^{\prime}}\right)\right)^{d+\epsilon_{1}}}
\end{aligned}
$$

where $\epsilon_{1}$ can be any positive number in $(0, \theta)$.

From this, it follows that

$$
\begin{aligned}
& \left\{\sum_{\alpha} \sum_{\tau \in I_{0}} \sum_{\nu=1}^{N(0, \tau)} \mu\left(Q_{\tau}^{0, \nu}\right)\left[m_{Q_{\tau}^{0, \nu}}\left(\left|E_{2}\right|\right)\right]^{p}\right\}^{1 / p} \\
& \leq C\left\{\sum _ { \tau \in I _ { 0 } } \sum _ { \nu = 1 } ^ { N ( 0 , \tau ) } \mu ( Q _ { \tau } ^ { 0 , \nu } ) \left[\sum_{k^{\prime}=1}^{\infty} \sum_{\tau^{\prime} \in I_{k^{\prime}}} \sum_{\nu^{\prime}=1}^{N\left(k^{\prime}, \tau^{\prime}\right)} 2^{-k^{\prime} \epsilon_{1}} \mu\left(Q_{\tau^{\prime}}^{k^{\prime}, \nu^{\prime}}\right)\left|D_{k^{\prime}}(f)\left(y_{\tau^{\prime}}^{k^{\prime}, \nu^{\prime}}\right)\right|\right.\right. \\
& \left.\left.\times \frac{1}{\left(1+\rho\left(y_{\tau}^{0, \nu}, y_{\tau^{\prime}}^{k^{\prime}, \nu^{\prime}}\right)\right)^{d+\epsilon_{1}}} \frac{1}{\mu\left(Q_{\tau}^{0, \nu}\right)} \int_{Q_{\tau}^{0, \nu}} \sum_{\alpha} \chi_{B\left(z_{\alpha}, A\left(C_{1}+C_{4}\right)\right)}(x) d \mu(x)\right]^{p}\right\}^{1 / p} \\
& \leq C\left\{\sum _ { \tau \in I _ { 0 } } \sum _ { \nu = 1 } ^ { N ( 0 , \tau ) } \mu ( Q _ { \tau } ^ { 0 , \nu } ) \left[\sum_{k^{\prime}=1}^{\infty} \sum_{\tau^{\prime} \in I_{k^{\prime}}} \sum_{\nu^{\prime}=1}^{N\left(k^{\prime}, \tau^{\prime}\right)} 2^{-k^{\prime} \epsilon_{1}} \mu\left(Q_{\tau^{\prime}}^{k^{\prime}, \nu^{\prime}}\right)\left|D_{k^{\prime}}(f)\left(y_{\tau^{\prime}}^{k^{\prime}, \nu^{\prime}}\right)\right|\right.\right. \\
& \left.\left.\times \frac{1}{\left(1+\rho\left(y_{\tau}^{0, \nu}, y_{\tau^{\prime}}^{k^{\prime}, \nu^{\prime}}\right)\right)^{d+\epsilon_{1}}}\right]^{p}\right\}^{1 / p} \\
& \leq C\left\{\sum _ { \tau \in I _ { 0 } } \sum _ { \nu = 1 } ^ { N ( 0 , \tau ) } \int _ { X } \chi _ { Q _ { \tau } ^ { 0 , \nu } } ( x ) \left[\sum_{k^{\prime}=1}^{\infty} \sum_{\tau^{\prime} \in I_{k^{\prime}}} \sum_{\nu^{\prime}=1}^{N\left(k^{\prime}, \tau^{\prime}\right)} 2^{-k^{\prime} \epsilon_{1}} \mu\left(Q_{\tau^{\prime}}^{k^{\prime}, \nu^{\prime}}\right)\left|D_{k^{\prime}}(f)\left(y_{\tau^{\prime}}^{k^{\prime}, \nu^{\prime}}\right)\right|\right.\right. \\
& \left.\left.\times \frac{1}{\left(1+\rho\left(x, y_{\tau^{\prime}}^{k^{\prime}, \nu^{\prime}}\right)\right)^{d+\epsilon_{1}}}\right]^{p} d \mu(x)\right\}^{1 / p} \\
& \text { (by } \left.\rho\left(x, y_{\tau^{\prime}}^{k^{\prime}, \nu^{\prime}}\right) \sim \rho\left(y_{\tau}^{0, \nu}, y_{\tau^{\prime}}^{k^{\prime}, \nu^{\prime}}\right) \text { for } x \in Q_{\tau}^{0, \nu}\right) \\
& \leq C\left\{\int _ { X } \left[\sum _ { k ^ { \prime } = 1 } ^ { \infty } 2 ^ { k ^ { \prime } ( d / r - \epsilon _ { 1 } ) } \left(M \left[\sum_{\tau^{\prime} \in I_{k^{\prime}}} \sum_{\nu^{\prime}=1}^{N\left(k^{\prime}, \tau^{\prime}\right)} \mu\left(Q_{\tau^{\prime}}^{k^{\prime}, \nu^{\prime}}\right)^{r}\right.\right.\right.\right.
\end{aligned}
$$




$$
\begin{aligned}
& \left.\left.\left.\left.\times\left|D_{k^{\prime}}(f)\left(y_{\tau^{\prime}}^{k^{\prime}, \nu^{\prime}}\right)\right|^{r} \chi_{Q_{\tau^{\prime}}^{k^{\prime}, \nu^{\prime}}}\right](x)\right)^{1 / r}\right]^{p} d \mu(x)\right\}^{1 / p} \quad \text { (by Lemma 2 } \\
= & C\left\{\int _ { X } \left[\sum _ { k ^ { \prime } = 1 } ^ { \infty } 2 ^ { k ^ { \prime } ( d / r - \epsilon _ { 1 } - d - s ) } \left(M \left[\sum_{\tau^{\prime} \in I_{k^{\prime}}} \sum_{\nu^{\prime}=1}^{N\left(k^{\prime}, \tau^{\prime}\right)} 2^{k s r}\right.\right.\right.\right. \\
& \left.\left.\left.\left.\times\left|D_{k^{\prime}}(f)\left(y_{\tau^{\prime}}^{k^{\prime}, \nu^{\prime}}\right)\right|^{r} \chi_{Q_{\tau^{\prime}}^{k^{\prime}, \nu^{\prime}}}\right](x)\right)^{1 / r}\right]^{p} d \mu(x)\right\}^{1 / p} \\
\leq & C\left\|\left\{\sum_{k^{\prime}=1}^{\infty}\left(M\left[\sum_{\tau^{\prime} \in I_{k^{\prime}}} \sum_{\nu^{\prime}=1}^{N\left(k^{\prime}, \tau^{\prime}\right)} 2^{k s r}\left|D_{k^{\prime}}(f)\left(y_{\tau^{\prime}}^{k^{\prime}, \nu^{\prime}}\right)\right|^{r} \chi_{Q_{\tau^{\prime}}^{k^{\prime}, \nu^{\prime}}}\right]\right)^{q / r}\right\}^{1 / q}\right\|_{L^{p}(X)}
\end{aligned}
$$

(when $q>1$, by the Hölder inequality; when $q \leq 1$, by (7))

$$
\leq C\|f\|_{F_{p q}^{s}(X)} .
$$

where we choose $\epsilon_{1} \in(0, \theta)$ and $r \in(0,1]$ such that $d /\left(\epsilon_{1}+d+s\right)<r<\min (p, q)$, which is possible by the conditions of Theorem1.6, and in the last step, we have used the Fefferman-Stein vector-valued maximal inequality in [4].

Estimates (11) and (12) imply that

$$
\left\{\sum_{\alpha} \sum_{\tau \in I_{0}} \sum_{\nu=1}^{N(0, \tau)} \mu\left(Q_{\tau}^{0, \nu}\right)\left[m_{Q_{\tau}^{0, \nu}}\left(\left|D_{0}\left(\psi_{\alpha} f\right)\right|\right)\right]^{p}\right\}^{1 / p} \leq C\|f\|_{F_{p q}^{s}(X)} .
$$

Now, by (13) and Definition 1.5, to finish the proof of Theorem 1.6, we need to show

$$
\left\{\sum_{\alpha}\left\|\left\{\sum_{k=1}^{\infty}\left[2^{k s}\left|D_{k}\left(\psi_{\alpha} f\right)\right|\right]^{q}\right\}^{1 / q}\right\|_{L^{p}(X)}^{p}\right\}^{1 / p} \leq C\|f\|_{F_{p q}^{s}(X)} .
$$


To do so, by Lemma 2.1, we write

$$
\begin{aligned}
D_{k}\left(\psi_{\alpha} f\right)(x)= & \int_{X} D_{k}(x, y) \psi_{\alpha}(y) f(y) d \mu(y) \\
= & \int_{X} D_{k}(x, y) \psi_{\alpha}(y) f(y) d \mu(y) \chi_{B\left(z_{\alpha}, A\left(3 C_{1}+C_{4}\right)\right)}(x) \\
= & \left\{\sum_{\tau^{\prime} \in I_{0}} \sum_{\nu^{\prime}=1}^{N\left(0, \tau^{\prime}\right)} \mu\left(Q_{\tau^{\prime}}^{0, \nu^{\prime}}\right) m_{Q_{\tau^{\prime}}^{0, \nu^{\prime}}}\left(D_{0}(f)\right) \int_{X} D_{k}(x, y) \psi_{\alpha}(y) \widetilde{D}_{Q_{\tau^{\prime}}^{0, \nu^{\prime}}}(y) d \mu(y)\right. \\
& +\sum_{k^{\prime}=1}^{\infty} \sum_{\tau^{\prime} \in I_{k^{\prime}}} \sum_{\nu^{\prime}=1}^{N\left(k^{\prime}, \tau^{\prime}\right)} \mu\left(Q_{\tau^{\prime}}^{k^{\prime}, \nu^{\prime}}\right) D_{k^{\prime}}(f)\left(y_{\tau^{\prime}}^{k^{\prime}, \nu^{\prime}}\right) \\
& \left.\times \int_{X} D_{k}(x, y) \psi_{\alpha}(y) \widetilde{D}_{k^{\prime}}\left(y, y_{\tau^{\prime}}^{k^{\prime}, \nu^{\prime}}\right) d \mu(y)\right\} \chi_{B\left(z_{\alpha}, A\left(3 C_{1}+C_{4}\right)\right)}(x) \\
= & \left(G_{1}+G_{2}\right) \chi_{B\left(z_{\alpha}, A\left(3 C_{1}+C_{4}\right)\right)}(x) .
\end{aligned}
$$

To estimate $G_{1}$, by the vanishing moment condition of $D_{k}$, we have

$$
\begin{aligned}
\mid \int_{X} & D_{k}(x, y) \psi_{\alpha}(y) \widetilde{D}_{Q_{\tau^{\prime}}^{0, \nu^{\prime}}}(y) d \mu(y) \mid \\
= & \left|\int_{X} D_{k}(x, y)\left[\psi_{\alpha}(y) \widetilde{D}_{Q_{\tau^{\prime}}^{0, \nu^{\prime}}}(y)-\psi_{\alpha}(x) \widetilde{D}_{Q_{\tau^{\prime}}^{0, \nu^{\prime}}}(x)\right] d \mu(y)\right| \\
\leq & \int_{\left\{y \in X: \rho(x, y) \leq \frac{1}{2 A}\left(6 A C_{1} 2^{-k}+\rho\left(x, y_{\tau^{\prime}}^{0, \nu^{\prime}}\right)\right)\right\}} \mid D_{k}(x, y)\left(\left[\psi_{\alpha}(y)-\psi_{\alpha}(x)\right] \widetilde{D}_{Q_{\tau^{\prime}}^{0, \nu^{\prime}}}(y)\right. \\
& \left.+\psi_{\alpha}(x)\left[\widetilde{D}_{Q_{\tau^{\prime}}^{0, \nu^{\prime}}}(y)-\widetilde{D}_{Q_{\tau^{\prime}}^{0, \nu^{\prime}}}(x)\right]\right) \mid d \mu(y) \\
\leq & C \int_{\left\{y \in X: \rho(x, y) \leq \frac{1}{2 A}\left(6 A C_{1} 2^{-k}+\rho\left(x, y_{\tau^{\prime}}^{0, \nu^{\prime}}\right)\right)\right\}}\left[\left|D_{k}(x, y)\right| \rho(x, y)^{\theta}\left|\widetilde{D}_{Q_{\tau^{\prime}}^{0, \nu^{\prime}}}(y)\right|\right. \\
& \left.+\rho(x, y)^{\epsilon} \frac{1}{\left(1+\rho\left(x, y_{\tau^{\prime}}^{0, \nu^{\prime}}\right)\right)^{d+2 \epsilon}}\right] d \mu(y) \\
\leq & C 2^{-k \epsilon} \frac{1}{\left(1+\rho\left(x, y_{\tau^{\prime}}^{0, \nu^{\prime}}\right)\right)^{d+\epsilon}},
\end{aligned}
$$

where $\epsilon$ can be any positive number in $(0, \theta)$ and will be determined later. From this, 
it follows that

$$
\begin{aligned}
& \left(\sum_{\alpha}\left\|\left\{\sum_{k=1}^{\infty}\left[2^{k s}\left|G_{1}\right| \chi_{B\left(z_{\alpha}, A\left(3 C_{1}+C_{4}\right)\right)}\right]^{q}\right\}^{1 / q}\right\|_{L^{p}(X)}^{p}\right)^{1 / p} \\
& \leq\left(\left\|\sum_{\alpha} \chi_{B\left(z_{\alpha}, A\left(3 C_{1}+C_{4}\right)\right)}\left\{\sum_{k=1}^{\infty}\left[2^{k s}\left|G_{1}\right|\right]^{q}\right\}^{1 / q}\right\|_{L^{p}(X)}^{p}\right)^{1 / p} \quad \text { (by (a)) } \\
& \leq C\left\{\left\|\left[\sum_{k=1}^{\infty} 2^{k s q}\left|G_{1}\right|^{q}\right]^{1 / q}\right\|_{L^{p}(X)}^{p}\right\}^{1 / p} \quad(\text { by }(9)) \\
& \leq C\left\{\int _ { X } [ \sum _ { k = 1 } ^ { \infty } 2 ^ { k ( s - \theta ) q } ] ^ { p / q } \left[\sum_{\tau^{\prime} \in I_{0}} \sum_{\nu^{\prime}=1}^{N\left(0, \tau^{\prime}\right)} \mu\left(Q_{\tau^{\prime}}^{0, \nu^{\prime}}\right) m_{Q_{\tau^{\prime}}^{0, \nu^{\prime}}}\left(\left|D_{0}(f)\right|\right)\right.\right. \\
& \left.\left.\times \frac{1}{\left(1+\rho\left(x, y_{\tau^{\prime}}^{0, \nu^{\prime}}\right)\right)^{d+\epsilon}}\right]^{p} d \mu(x)\right\}^{1 / p} \\
& \leq C\left\{\int_{X}\left[\sum_{\tau^{\prime} \in I_{0}} \sum_{\nu^{\prime}=1}^{N\left(0, \tau^{\prime}\right)} \mu\left(Q_{\tau^{\prime}}^{0, \nu^{\prime}}\right) m_{Q_{\tau^{\prime}}^{0, \nu^{\prime}}}\left(\left|D_{0}(f)\right|\right) \frac{1}{\left(1+\rho\left(x, y_{\tau^{\prime}}^{0, \nu^{\prime}}\right)\right)^{d+\epsilon}}\right]^{p} d \mu(x)\right\}^{1 / p} \\
& \int\left\{\sum_{\tau^{\prime} \in I_{0}} \sum_{\nu^{\prime}=1}^{N\left(0, \tau^{\prime}\right)} \mu\left(Q_{\tau^{\prime}}^{0, \nu^{\prime}}\right)\left[m_{Q_{\tau^{\prime}}^{0, \nu^{\prime}}}\left(\left|D_{0}(f)\right|\right)\right]^{p}\right. \\
& \left.\times \int_{X} \frac{1}{\left(1+\rho\left(x, y_{\tau^{\prime}}^{0, \nu^{\prime}}\right)\right)^{(d+\epsilon) p}} d \mu(x)\right\}^{1 / p}, p \leq 1 \\
& \text { (by }(7) \text { and } \mu\left(Q_{\tau^{\prime}}^{0, \nu^{\prime}}\right) \sim C \text { ) } \\
& \leq C\left\{\left\{\int_{X}\left(\sum_{\tau^{\prime} \in I_{0}} \sum_{\nu^{\prime}=1}^{N\left(0, \tau^{\prime}\right)} \mu\left(Q_{\tau^{\prime}}^{0, \nu^{\prime}}\right)\left[m_{Q_{\tau^{\prime}}^{0, \nu^{\prime}}}\left(\left|D_{0}(f)\right|\right)\right]^{p} \frac{1}{\left(1+\rho\left(x, y_{\tau^{\prime}}^{0, \nu^{\prime}}\right)\right)^{d+\epsilon}}\right)\right.\right. \\
& \left.\times\left[\int_{X} \frac{1}{(1+\rho(x, y))^{d+\epsilon}} d \mu(y)\right]^{p / p^{\prime}} d \mu(x)\right\}^{1 / p}, 1<p<\infty \\
& \text { (by the Hölder inequality) } \\
& \leq C\left\{\sum_{\tau^{\prime} \in I_{0}} \sum_{\nu^{\prime}=1}^{N\left(0, \tau^{\prime}\right)} \mu\left(Q_{\tau^{\prime}}^{0, \nu^{\prime}}\right)\left[m_{Q_{\tau^{\prime}}^{0, \nu^{\prime}}}\left(\left|D_{0}(f)\right|\right)\right]^{p}\right\}^{1 / p} \leq C\|f\|_{F_{p q}^{s}(X)},
\end{aligned}
$$


which is a desired estimate for $G_{1}$, where we choose $\epsilon \in(0, \theta)$ such that $p>d /(d+\epsilon)$.

To estimate the terms relative to $G_{2}$, we first establish the following estimate

$$
\begin{aligned}
\left|\int_{X} D_{k}(x, y) \psi_{\alpha}(y) \widetilde{D}_{k^{\prime}}\left(y, y_{\tau^{\prime}}^{k^{\prime}, \nu^{\prime}}\right) d \mu(y)\right| & \\
& \leq C 2^{-\left|k-k^{\prime}\right| \epsilon} \frac{2^{-\left(k \wedge k^{\prime}\right) \epsilon}}{\left(2^{-\left(k \wedge k^{\prime}\right)}+\rho\left(x, y_{\tau^{\prime}}^{k^{\prime}, \nu^{\prime}}\right)\right)^{d+\epsilon}},
\end{aligned}
$$

where $a \wedge b=\min (a, b)$ for any $a, b \in \mathbb{R}$ and $\epsilon$ can be any positive number in $(0, \theta)$. Let us first consider the case $k \geq k^{\prime}$ of (16). In this case, by the vanishing moment of $D_{k}$, we can write

$$
\begin{aligned}
\mid \int_{X} & D_{k}(x, y) \psi_{\alpha}(y) \widetilde{D}_{k^{\prime}}\left(y, y_{\tau^{\prime}}^{k^{\prime}, \nu^{\prime}}\right) d \mu(y) \mid \\
= & \left|\int_{X} D_{k}(x, y)\left[\psi_{\alpha}(y) \widetilde{D}_{k^{\prime}}\left(y, y_{\tau^{\prime}}^{k^{\prime}, \nu^{\prime}}\right)-\psi_{\alpha}(x) \widetilde{D}_{k^{\prime}}\left(x, y_{\tau^{\prime}}^{k^{\prime}, \nu^{\prime}}\right)\right] d \mu(y)\right| \\
= & \mid \int_{X} D_{k}(x, y)\left(\left[\psi_{\alpha}(y)-\psi_{\alpha}(x)\right] \widetilde{D}_{k^{\prime}}\left(y, y_{\tau^{\prime}}^{k^{\prime}, \nu^{\prime}}\right)\right. \\
& \left.+\psi_{\alpha}(x)\left[\widetilde{D}_{k^{\prime}}\left(y, y_{\tau^{\prime}}^{k^{\prime}, \nu^{\prime}}\right)-\widetilde{D}_{k^{\prime}}\left(x, y_{\tau^{\prime}}^{k^{\prime}, \nu^{\prime}}\right)\right]\right) d \mu(y) \mid \\
\leq & C 2^{-k \theta} \int_{X}\left|D_{k}(x, y)\right|\left|\widetilde{D}_{k^{\prime}}\left(y, y_{\tau^{\prime}}^{k^{\prime}, \nu^{\prime}}\right)\right| d \mu(y) \\
& +C \int_{X}\left|D_{k}(x, y)\right| \frac{2^{-k \epsilon} \rho(x, y)^{\epsilon}}{\left(2^{-k^{\prime}}+\rho\left(x, y_{\tau^{\prime}}^{k^{\prime}, \nu^{\prime}}\right)\right)^{d+2 \epsilon}} d \mu(y) \\
\leq & C 2^{-\left(k-k^{\prime}\right) \epsilon} \frac{2^{-k^{\prime} \epsilon}}{\left(2^{-k^{\prime}}+\rho\left(x, y_{\tau^{\prime}}^{k^{\prime}, \nu^{\prime}}\right)\right)^{d+\epsilon}}
\end{aligned}
$$

which is a desired estimate. We now consider the case $k<k^{\prime}$ of (16). In this case, by the vanishing moment of $\widetilde{D}_{k^{\prime}}$, we have

$$
\begin{aligned}
\mid \int_{X} & D_{k}(x, y) \psi_{\alpha}(y) \widetilde{D}_{k^{\prime}}\left(y, y_{\tau^{\prime}}^{k^{\prime}, \nu^{\prime}}\right) d \mu(y) \mid \\
= & \left|\int_{X}\left[D_{k}(x, y) \psi_{\alpha}(y)-D_{k}\left(x, y_{\tau^{\prime}}^{k^{\prime}, \nu^{\prime}}\right) \psi_{\alpha}\left(y_{\tau^{\prime}}^{k^{\prime}, \nu^{\prime}}\right)\right] \widetilde{D}_{k^{\prime}}\left(y, y_{\tau^{\prime}}^{k^{\prime}, \nu^{\prime}}\right) d \mu(y)\right| \\
\leq & \int_{\left\{y \in X: \rho\left(y, y_{\tau^{\prime}}^{k^{\prime}, \nu^{\prime}}\right) \leq \frac{1}{2 A}\left(2^{-k}+\rho\left(x, y_{\tau^{\prime}}^{k^{\prime}, \nu^{\prime}}\right)\right)\right\}} \mid\left[D_{k}(x, y) \psi_{\alpha}(y)-D_{k}\left(x, y_{\tau^{\prime}}^{k^{\prime}, \nu^{\prime}}\right) \psi_{\alpha}\left(y_{\tau^{\prime}}^{k^{\prime}, \nu^{\prime}}\right)\right] \\
& \times \widetilde{D}_{k^{\prime}}\left(y, y_{\tau^{\prime}}^{k^{\prime}, \nu^{\prime}}\right) \mid d \mu(y)+\int_{\left\{y \in X: \rho\left(y, y_{\tau^{\prime}}^{k^{\prime}, \nu^{\prime}}\right)>\frac{1}{2 A}\left(2^{-k}+\rho\left(x, y_{\tau^{\prime}}^{k^{\prime}, \nu^{\prime}}\right)\right)\right\}} \ldots \\
= & H_{1}+H_{2} .
\end{aligned}
$$


On $H_{1}$, from the support of $D_{k}$ and the regularities of $D_{k}$ and $\psi_{\alpha}$, it follows that

$$
\begin{aligned}
H_{1} \leq & C\left\{2^{k\left(d+\epsilon_{1}\right)} \chi_{B\left(y_{\tau^{\prime}}^{k^{\prime}, \nu^{\prime}}, C 2^{-k}\right)}(x)+\frac{2^{-k \theta}}{\left(2^{-k}+\rho\left(x, y_{\tau^{\prime}}^{k^{\prime}, \nu^{\prime}}\right)\right)^{d+\theta}}\right\} \\
& \times \int_{X} \rho\left(y, y_{\tau^{\prime}}^{k^{\prime}, \nu^{\prime}}\right)^{\epsilon_{1}}\left|\widetilde{D}_{k^{\prime}}\left(y, y_{\tau^{\prime}}^{k^{\prime}, \nu^{\prime}}\right)\right| d \mu(y) \\
\leq & C\left\{2^{k\left(d+\epsilon_{1}\right)} \chi_{B\left(y_{\tau^{\prime}}^{k^{\prime}, \nu^{\prime}}, C 2^{-k}\right)}(x)+\frac{2^{-k \theta}}{\left(2^{-k}+\rho\left(x, y_{\tau^{\prime}}^{k^{\prime}, \nu^{\prime}}\right)\right)^{d+\theta}}\right\} \\
& \times \int_{X} \rho\left(y, y_{\tau^{\prime}}^{k^{\prime}, \nu^{\prime}}\right)^{\epsilon_{1}} \frac{2^{-k^{\prime} \epsilon}}{\left(2^{-k^{\prime}}+\rho\left(y, y_{\tau^{\prime}}^{k^{\prime}, \nu^{\prime}}\right)\right)^{d+\epsilon}} d \mu(y) \\
\leq & C 2^{-\left(k^{\prime}-k\right) \epsilon_{1}} \frac{2^{-k \epsilon_{1}}}{\left(2^{-k}+\rho\left(x, y_{\tau^{\prime}}^{k^{\prime}, \nu^{\prime}}\right)\right)^{d+\epsilon_{1}}},
\end{aligned}
$$

where $\epsilon$ and $\epsilon_{1}$ can be any positive number, respectively, in $(0, \theta)$ and in $(0, \epsilon)$. This is a desired estimate for $H_{1}$. For $H_{2}$, by the size conditions of $D_{k}$ and $\widetilde{D}_{k^{\prime}}$, we obtain

$$
\begin{aligned}
H_{2} & \leq C \frac{2^{-k^{\prime} \epsilon}}{\left(2^{-k}+\rho\left(x, y_{\tau^{\prime}}^{k^{\prime}, \nu^{\prime}}\right)\right)^{d+\epsilon}} \int_{X}\left|D_{k}(x, y) \psi_{\alpha}(y)-D_{k}\left(x, y_{\tau^{\prime}}^{k^{\prime}, \nu^{\prime}}\right) \psi_{\alpha}\left(y_{\tau^{\prime}}^{k^{\prime}, \nu^{\prime}}\right)\right| d \mu(y) \\
& \leq C 2^{-\left(k^{\prime}-k\right) \epsilon} \frac{2^{-k \epsilon}}{\left(2^{-k}+\rho\left(x, y_{\tau^{\prime}}^{k^{\prime}, \nu^{\prime}}\right)\right)^{d+\epsilon}},
\end{aligned}
$$

where $\epsilon$ can be any positive number in $(0, \theta)$. This is also a desired estimate for $H_{2}$. Thus, (16) holds.

From (16), it follows that

$$
\begin{aligned}
& \left(\sum_{\alpha}\left\|\left\{\sum_{k=1}^{\infty}\left[2^{k s}\left|G_{2}\right| \chi_{B\left(z_{\alpha}, A\left(3 C_{1}+C_{4}\right)\right)}\right]^{q}\right\}^{1 / q}\right\|_{L^{p}(X)}^{p}\right)^{1 / p} \\
& \leq C\left(\int _ { X } \sum _ { \alpha } \chi _ { B ( z _ { \alpha } , A ( 3 C _ { 1 } + C _ { 4 } ) ) } ( x ) \left\{\sum _ { k = 1 } ^ { \infty } 2 ^ { k s q } \left[\sum_{k^{\prime}=1}^{\infty} \sum_{\tau^{\prime} \in I_{k^{\prime}}} \sum_{\nu^{\prime}=1}^{N\left(k^{\prime}, \tau^{\prime}\right)} \mu\left(Q_{\tau^{\prime}}^{k^{\prime}, \nu^{\prime}}\right)\right.\right.\right. \\
& \left.\left.\left.\times\left|D_{k^{\prime}}(f)\left(y_{\tau^{\prime}}^{k^{\prime}, \nu^{\prime}}\right)\right| 2^{-\left|k-k^{\prime}\right| \epsilon_{1}} \frac{2^{-\left(k \wedge k^{\prime}\right) \epsilon_{1}}}{\left(2^{-\left(k \wedge k^{\prime}\right)}+\rho\left(x, y_{\tau^{\prime}}^{k^{\prime}, \nu^{\prime}}\right)\right)^{d+\epsilon_{1}}}\right]^{q}\right\}^{p / q} d \mu(x)\right)^{1 / p}
\end{aligned}
$$

(by (a)) 


$$
\begin{aligned}
\leq & C\left(\int _ { X } \left\{\sum _ { k = 1 } ^ { \infty } 2 ^ { k s q } \left[\sum_{k^{\prime}=1}^{\infty} 2^{\left(k \wedge k^{\prime}\right) d-\left|k-k^{\prime}\right| \epsilon_{1}-k^{\prime}(s+d)+\left[k^{\prime}-\left(k \wedge k^{\prime}\right)\right] d / r}\right.\right.\right. \\
& \left.\left.\left.\times\left(M\left[\sum_{\tau^{\prime} \in I_{k^{\prime}}} \sum_{\nu^{\prime}=1}^{N\left(k^{\prime}, \tau^{\prime}\right)} 2^{k^{\prime} s r}\left|D_{k^{\prime}}(f)\left(y_{\tau^{\prime}}^{k^{\prime}, \nu^{\prime}}\right)\right|^{r} \chi_{Q_{\tau^{\prime}}^{k^{\prime}, \nu^{\prime}}}\right](x)\right)^{1 / r}\right]^{q}\right\}^{p / q} d \mu(x)\right)^{1 / p}
\end{aligned}
$$

(by Lemma 2.2)

$$
\begin{aligned}
\leq & C\left(\int _ { X } \left\{\sum _ { k = 1 } ^ { \infty } \left[\sum _ { k ^ { \prime } = 1 } ^ { k } 2 ^ { ( k - k ^ { \prime } ) ( s - \epsilon _ { 1 } ) } \left(M \left[\sum_{\tau^{\prime} \in I_{k^{\prime}}} \sum_{\nu^{\prime}=1}^{N\left(k^{\prime}, \tau^{\prime}\right)} 2^{k^{\prime} s r}\right.\right.\right.\right.\right. \\
& \left.\left.\left.\left.\left.\times\left|D_{k^{\prime}}(f)\left(y_{\tau^{\prime}}^{k^{\prime}, \nu^{\prime}}\right)\right|^{r} \chi_{Q_{\tau^{\prime}}^{k^{\prime}, \nu^{\prime}}}\right](x)\right)^{1 / r}\right]^{q}\right\}^{p / q} d \mu(x)\right)^{1 / p} \\
& +C\left(\int _ { X } \left\{\sum _ { k = 1 } ^ { \infty } \left[\sum _ { k ^ { \prime } = k + 1 } ^ { \infty } 2 ^ { ( k - k ^ { \prime } ) [ s + \epsilon _ { 1 } + d ( 1 - 1 / r ) ] } \left(M \left[\sum_{\tau^{\prime} \in I_{k^{\prime}}} \sum_{\nu^{\prime}=1}^{N\left(k^{\prime}, \tau^{\prime}\right)} 2^{k^{\prime} s r}\right.\right.\right.\right.\right. \\
& \left.\left.\left.\left.\left.\times\left|D_{k^{\prime}}(f)\left(y_{\tau^{\prime}}^{k^{\prime}, \nu^{\prime}}\right)\right|^{r} \chi_{Q_{\tau^{\prime}}} k^{k^{\prime}, \nu^{\prime}}\right](x)\right)^{1 / r}\right]^{q}\right\}^{p / q} d \mu(x)\right)^{1 / p} \\
\leq & C\left\{\int_{X}\left[\sum_{k^{\prime}=1}^{\infty}\left(M\left[\sum_{\tau^{\prime} \in I_{k^{\prime}}} \sum_{\nu^{\prime}=1}^{N\left(k^{\prime}, \tau^{\prime}\right)} 2^{k^{\prime} s r}\left[\left.\begin{array}{l}
1 / p \\
\times
\end{array} D_{k^{\prime}}(f)\left(y_{\tau^{\prime}}^{k^{\prime}, \nu^{\prime}}\right)\right|^{r} \chi_{Q_{\tau^{\prime}}^{k^{\prime}, \nu^{\prime}}}\right](x)\right)^{q / r}\right]^{p / q} d \mu(x)\right)^{1 / q}\right.
\end{aligned}
$$

(by (7), when $q \leq 1$; by the Hölder inequality, when $q>1$ )

$$
\leq C\left\|\left\{\sum_{k^{\prime}=1}^{\infty}\left(\sum_{\tau^{\prime} \in I_{k^{\prime}}} \sum_{\nu^{\prime}=1}^{N\left(k^{\prime}, \tau^{\prime}\right)} 2^{k^{\prime} s q}\left|D_{k^{\prime}}(f)\left(y_{\tau^{\prime}}^{k^{\prime}, \nu^{\prime}}\right)\right|^{q} \chi_{Q_{\tau^{\prime}}^{k^{\prime}, \nu^{\prime}}}\right)\right\}^{1 / q}\right\|_{L^{p}(X)}
$$

(by the Fefferman-Stein vector-valued inequality in [4]) 


$$
\leq C\left\|\left\{\sum_{k^{\prime}=1}^{\infty} 2^{k^{\prime} s q}\left|D_{k^{\prime}}(f)\right|^{q}\right\}^{1 / q}\right\|_{L^{p}(X)}=C\|f\|_{F_{p q}^{s}(X)},
$$

where, in the second-to-last inequality, we have used the arbitrariness of $y_{\tau^{\prime}}^{k^{\prime}, \nu^{\prime}}$, and we choose $\epsilon_{1}>0$ and $r \in(0,1]$ such that $\epsilon_{1}>s$ and $d /\left(d+s+\epsilon_{1}\right)<r<\min (p, q)$.

Estimates (15) and (17) imply (14). By combining Definition 1.5, (13) with (14), we have finished the proof of Theorem 1.6.

Acknowledgements. Part of this paper was written while the author visited the Friedrich-Schiller University at Jena as a Humboldt research fellow. He gratefully acknowledges the support of the Alexander von Humboldt Foundation in Germany. Special thanks are due to Professor Hans Triebel for him to propose this problem to the author and to share his many ideas with the author. The author is also partially supported by NNSF (No. 10271015) and RFDP (No. 20020027004) of China.

\section{References}

[1] M. Christ, $A T(b)$ theorem with remarks on analytic capacity and the Cauchy integral, Colloq. Math. 60/61 (1990), 601-628.

[2] Ronald R. Coifman and G. Weiss, Analyse harmonique non-commutative sur certains espaces homogènes, Springer-Verlag, Berlin, 1971, Étude de certaines intégrales singulières; Lecture Notes in Mathematics, Vol. 242.

[3] G. David, J.-L. Journé, and S. Semmes, Opérateurs de Calderón-Zygmund, fonctions paraaccrétives et interpolation, Rev. Mat. Iberoamericana 1 (1985), 1-56.

[4] C. Fefferman and E. M. Stein, Some maximal inequalities, Amer. J. Math. 93 (1971), 107-115.

[5] M. Frazier and Björn Jawerth, A discrete transform and decompositions of distribution spaces, J. Funct. Anal. 93 (1990), 34-170.

[6] Y. Han, Inhomogeneous Calderón reproducing formula on spaces of homogeneous type, J. Geom. Anal. 7 (1997), 259-284.

[7] Y. Han, S. Lu, and D. Yang, Inhomogeneous Besov and Triebel-Lizorkin spaces on spaces of homogeneous type, Approx. Theory Appl. (N.S.) 15 (1999), 37-65.

[8] __ Inhomogeneous Triebel-Lizorkin spaces on spaces of homogeneous type, Math. Sci. Res. Hot-Line 3 (1999), 1-29.

[9] _ Inhomogeneous discrete Calderón reproducing formulas for spaces of homogeneous type, J. Fourier Anal. Appl. 7 (2001), 571-600.

[10] Y. Han and E. T. Sawyer, Littlewood-Paley theory on spaces of homogeneous type and the classical function spaces, Mem. Amer. Math. Soc. 110 (1994), 1-126.

[11] Y. Han and D. Yang, New characterizations and applications of inhomogeneous Besov and Triebel-Lizorkin spaces on homogeneous type spaces and fractals, Dissertationes Math. (Rozprawy Mat.) 403 (2002), 1-102.

[12] __ Some new spaces of Besov and Triebel-Lizorkin type on homogeneous spaces, Studia Math. 156 (2003), 67-97. 
[13] R. A. Macías and C. Segovia, Lipschitz functions on spaces of homogeneous type, Adv. in Math. 33 (1979), 257-270.

[14] H. Triebel, Theory of function spaces. II, Monographs in Mathematics, vol. 84, Birkhäuser Verlag, Basel, 1992, ISBN 3-7643-2639-5.

[15] _ Fractals and spectra, Monographs in Mathematics, vol. 91, Birkhäuser Verlag, Basel, 1997, ISBN 3-7643-5776-2, Related to Fourier analysis and function spaces.

[16] _ The structure of functions, Monographs in Mathematics, vol. 97, Birkhäuser Verlag, Basel, 2001, ISBN 3-7643-6546-3.

[17] _ Function spaces in Lipschitz domains and on Lipschitz manifolds. Characteristic functions as pointwise multipliers, Rev. Mat. Complut. 15 (2002), 475-524.

[18] D. Yang, Besov spaces and applications on homogeneous type spaces and fractals, Studia Math. 156 (2003), 15-30 